\title{
Development of a SPOC of Computer Ethics for students of Computer Science degree
}

\author{
Alicia García-Holgado \\ Computer Science Department, \\ GRIAL Research Group, IUCE \\ University of Salamanca \\ Salamanca, Spain \\ aliciagh@usal.es
}

\begin{abstract}
Andrea Vázquez-Ingelmo
Computer Science Department,

GRIAL Research Group, IUCE

University of Salamanca

Salamanca, Spain

andreavazquez@usal.es
\end{abstract}

Rosa María Gil Iranzo

Computer Science and Industrial

Engineering Department

Universitat de Lleida

Lleida, Spain

rgil@diei.udl.cat

\author{
Francisco J. García-Peñalvo \\ Computer Science Department, \\ GRIAL Research Group, IUCE \\ University of Salamanca \\ Salamanca, Spain \\ fgarcia@usal.es
}

Adriana Gamazo

Didactics, Organization and Research

Methods Department, GRIAL

GRIAL Research Group, IUCE

University of Salamanca

Salamanca, Spain

adrianagamazo@usal.es

\author{
Ismar Frango Silveira \\ Faculdade de Computação e \\ Informática \\ Universidade Presbiteriana Mackenzie \\ Sao Paulo, Brazil \\ ismarfrango@gmail.com
}

\author{
Roberto Therón \\ Computer Science Department, \\ GRIAL Research Group, IUCE \\ University of Salamanca \\ Salamanca, Spain \\ theron@usal.es
}

Carina S. González-González Computer and Systems Engineering Department, University Institute of Women's Studies

Universidad de La Laguna

San Cristóbal de La Laguna, Spain cjgonza@ull.edu.es

Marc Alier Forment

Department of Service and Information System Engineering, BCN-SEER

BarcelonaTech

Barcelona, Spain

marc.alier@upc.edu

\begin{abstract}
Technology brings different benefits to society and involves challenges and ethical dilemmas that must be considered during any technology development. In this sense, graduates must recognise the social, legal, ethical and cultural issues inherent to the discipline of computer science. However, there is a lack of integration of computer ethics in the computer science curriculum in Spanish universities. This work introduces a pilot experience to develop a Small Private Open Course (SPOC) to introduce computer ethics as an extracurricular activity in the Bachelor's Degree of Computer Engineering at the University of Salamanca.
\end{abstract}

Keywords-computer ethics, engineering, higher education, SPOC, innovation

\section{INTRODUCTION}

Technology plays a key role in today's society, a presence that has increased with the global crisis due to COVID-19. Artificial Intelligence algorithms to support decision-making, virtual reality in education, or robots in medical contexts are examples of how technology is part of a large number of processes. Technology brings different benefits and poses challenges and ethical dilemmas that must be considered when considering any development.

According to the Computer Science Curriculum 2013 developed by two of the leading associations in technology and engineering issues [1], computer science graduates should have a range of personal skills not only focused on technological issues, but also the social, legal, ethical and cultural issues inherent in the discipline of computer science.

Although the social and ethical aspects of technology have been included in some computer science curricula for more than twenty years, there is a wide variety among computer ethics courses in terms of the methodologies $[2,3]$ and how to incorporate them into the curriculum [4]. There is also a lack of integration of computer ethics in the Spanish context. If we look at the collaborative database on higher education courses or programmes that deal with "technological ethics" [5], the number of Spanish universities is much lower than in other countries.

In the Bachelor's Degree in Computer Science at the University of Salamanca, some courses deal with diversity and inclusion issues, but none of the courses are addressed from an ethical point of view. Likewise, there is no specific subject that focuses on the ethical aspects of computer science.

In this context, this work describes a pilot test carried out in the Bachelor's Degree in Computer Science at the University of Salamanca through a teaching innovation project funded in the 2020-21 academic year. The project has allowed us to generate a set of contents related to computer ethics and collect the participants' perception to improve the proposal in future courses. The pilot was conducted in the second term of the 2020-21 academic year in the course Software Engineering I, taught in the second year, and in the course Human-Computer Interaction, taught in the third year.

\section{OBJECTIVE}

The general objective of the innovation project is to incorporate ethical aspects related to software development in the Bachelor's Degree in Computer Science through a SPOC (Small Private Open Course). The implementation was carried out during the 2020-21 academic year.

The following specific objectives have been defined in order to achieve the general objective:

- Carry out the instructional design of the SPOC.

- Designing and recording online talks on different ethical aspects of software development as part of the courses Software Engineering I and Human-Computer Interaction.

- Create and implement the SPOC in the courses listed above. 


\section{SPOC CONTENTS}

The SPOC syllabus is divided into four main blocks: introduction to computer ethics, ethics and human-computer interaction, ethics and universal design, and ethics and artificial intelligence.

The video pills for each of the modules were created through a set of online talks. National and international experts gave the talks on ethical issues in computing. Each talk has a maximum duration of 40 minutes with a structure that allows for the later editing the recordings and creating the video lessons. Each talk is divided into three parts: presentation by the expert, questions, and a survey about the talk.

The talks were given in the following order throughout the second semester, taking into account the teaching activities of the targeted students (Fig. 1). This sequence is determined by the SPOC design, as it is organised into four modules, one associated with each talk:

- "Introduction to computer ethics" by Marc Alier Forment.

- "Ethics and Human-Computer Interaction" by Carina Soledad González González.

- "Ethics and Universal Design” by Ismar Frango.

- "Ethics and Artificial Intelligence" by Rosa María Gil Iranzo.

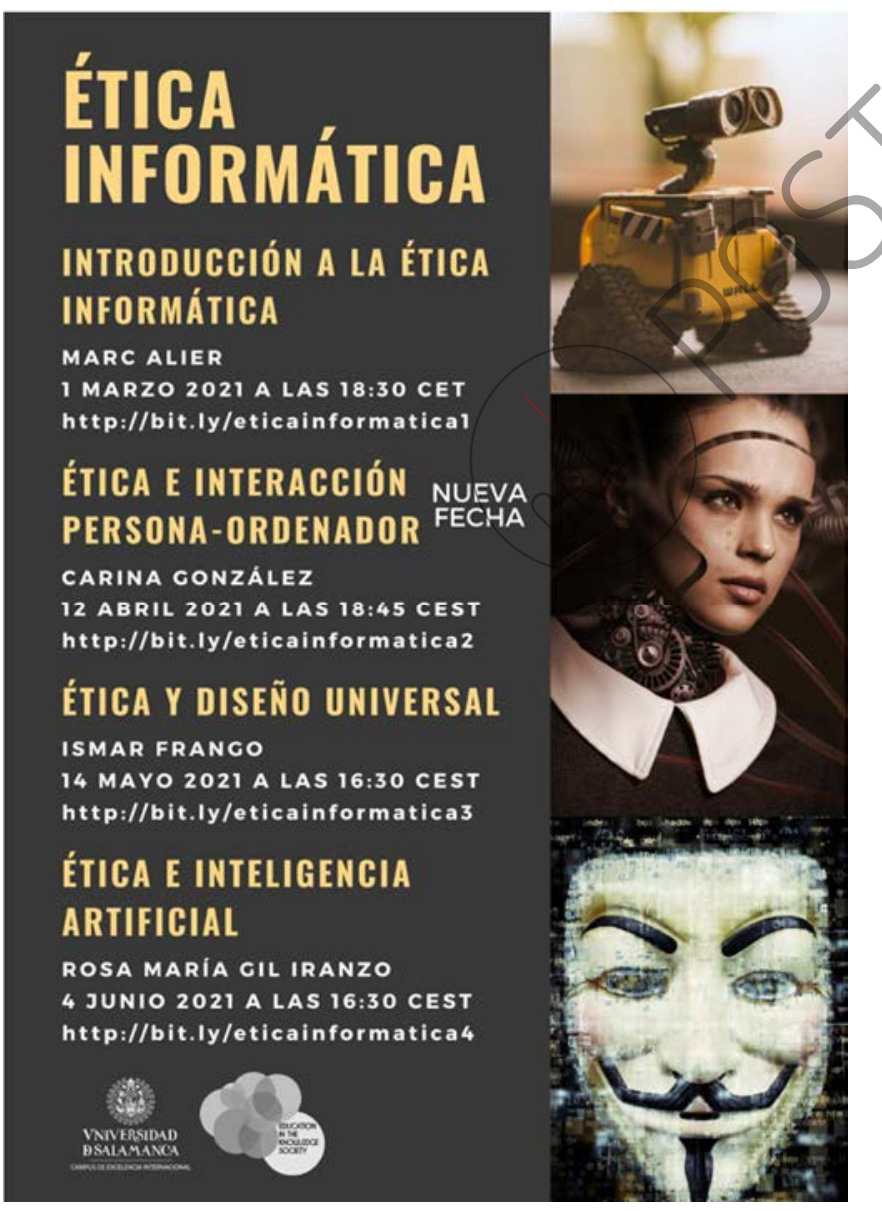

Fig. 1. Schedule of talks

\section{INTEGRATION IN THE TEACHING-LEARNING PROCESS}

The SPOC is a voluntary, non-evaluable activity related to the courses where the pilot was developed, but it is not carried out during academic hours. However, it was integrated with the two courses involved. The aim is to encourage participation in the SPOC by adding ethical aspects to the final projects carried out in each course. For this purpose, an indicator has been added to the rubrics used to evaluate these projects. This indicator was not associated with participation in the SPOC because it was a voluntary extracurricular activity.

Furthermore, we define some requirements in order to value student participation. In particular, it was established as a requirement to attend at least two talks and answer a final questionnaire on the perception of computer ethics to obtain the SPOC certificate of completion.

We also disseminate the project and each talk inside the virtual spaces of both courses. First, we created a video presentation about the talks adapted to each course, valorising the importance of ethics in computer science and explaining the new rubric indicator for the final project. Second, we shared the information about the talks and the recordings in the virtual campus of Software Engineering I and the Microsoft Teams space of the Human-Computer Interaction course.

\section{Results}

The number of students involved in the pilot through Software Engineering I and Human-Computer Interaction courses was 232. A total of 101 students attended talks (Table 1), with only 24 of them not belonging to the bachelor's degree of Computer Science. This figure indicates that the sample represents around $33 \%$ of the pilot population.

TABLE I. PARTICIPANTS IN THE TALKS

\begin{tabular}{lll}
\hline Talk & Registered & Attendees \\
\hline Introduction to computer ethics & 103 & 73 \\
\hline $\begin{array}{l}\text { Ethics and Human-Computer } \\
\text { Interaction }\end{array}$ & 97 & 51 \\
\hline Ethics and universal design & 79 & 44 \\
\hline Ethics and Artificial Intelligence & 70 & 38 \\
\hline
\end{tabular}

Regarding the percentage of students who have finally attended two or more talks, they represent $58.42 \%$ of the total number of attendees. Specifically, 59 students, of which 10 came from outside the degree.

Finally, concerning the satisfaction of the participants (Table 2), the attendees answered seven Likert items of five points:

Q1. Overall I consider the talk to was satisfactory.

Q2. I would recommend the talk to people with the same interests as me.

Q3. I would attend more talks by the same speaker.

Q4. I found the speaker's explanations clear and understandable.

Q5. The length of the talk was adequate.

Q6. I am interested in participating in similar activities on other topics.

Q7. The content of the talk was interesting. 
TABLE II. PARTICIPANTS IN THE SATISFACTION QUESTIONNAIRE

\begin{tabular}{lll}
\hline Talks Answers & Percentage \\
\hline Introduction to computer ethics & 72 & $98,63 \%$ \\
\hline $\begin{array}{l}\text { Ethics and Human-Computer } \\
\text { Interaction }\end{array}$ & 50 & $98,04 \%$ \\
\hline Ethics and universal design & 39 & $88,64 \%$ \\
\hline Ethics and Artificial Intelligence & 24 & $63,16 \%$ \\
\hline
\end{tabular}

In general terms, the participants' satisfaction is high in all the questionnaire items for all the talks (Fig. 2), with the average number of answers between 4 and 5 (agree and strongly agree). Analysing the frequency of responses for each question, the percentage of total responses with value 5 (strongly agree) for $\mathrm{N}=185$ ranges between $58.9 \%$ and $66.5 \%$ among the different talks.

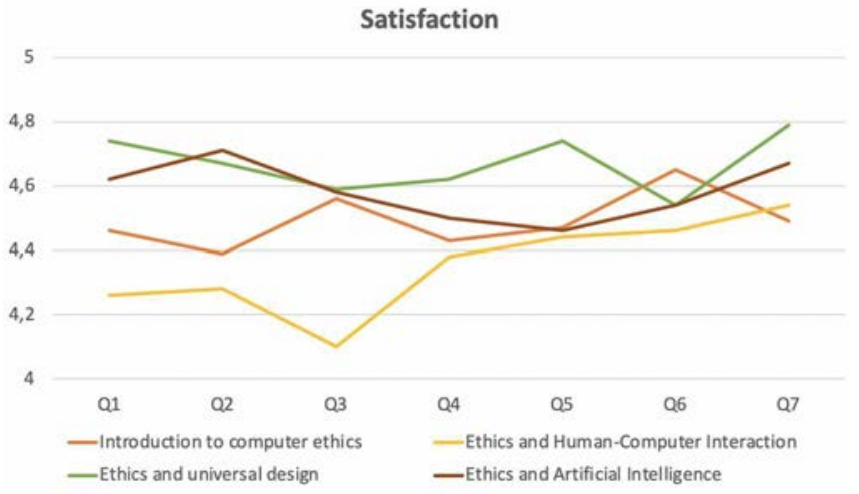

Fig. 2. Mean scores of the satisfaction questions for each talk

\section{CONCLUSIONS}

On the one hand, the innovation of this proposal lies in the incorporation of ethics in computer science as part of an official bachelor's degree, where there are no courses that deals with these concepts. On the other hand, an online approach was used to adapt to the different scenarios generated by the current health crisis [6-8], while at the same time generating a base of video content that is integrated into the SPOC. Finally, the SPOC will be available for being used in subsequent academic years, mainly for students of the bachelor's degree in Computer Science.

In terms of results, there has been a high level of student participation. The focus of the talks and the contents have been well received, although this figure could be increased in future academic years.

The online format of the talks has made it easier for students to participate during non-academic hours. In addition, this format has allowed access to national and international experts who otherwise would not have been able to participate in person due to a lack of budget.

Finally, the final result of the pilot has provided a SPOC with four modules consisting of a set of educational pills and evaluation activities. This result needs to be tested in future courses to determine whether this way of generating the SPOC is valid or whether the process itself is what should be replicated.

\section{ACKNOWLEDGMENT}

This work is part of the teaching innovation project "Design and creation of a SPOC on ethical considerations in software development for students of the Degree in Computer Science" (ID2020/002) funded by the University of Salamanca (Spain) in the academic year 2020-21; and partially supported by the Spanish Ministry of Science, Innovation and Universities through a FPU grant (FPU017/01252).

\section{REFERENCES}

[1] ACM and IEEE Computer Society, Computer Science Curricula 2013. 2013 .

[2] C. Fiesler, N. Garrett, and N. Beard, "What Do We Teach When We Teach Tech Ethics? A Syllabi Analysis," presented at the Proceedings of the 51st ACM Technical Symposium on Computer Science Education, Portland, OR, USA, 2020.

[3] O. Nasir, S. Muntaha, R. T. Javed, and J. Qadir, "Work in Progress: Pedagogy of Engineering Ethics: A Bibliometric and Curricular Analysis," in 2021 IEEE Global Engineering Education Conference (EDUCON), 21-23 April 2021 2021, pp. 1553-1557, doi: 10.1109/EDUCON46332.2021.9453923.

[4] M. J. Casañ, M. Alier, and A. Llorens, "Teaching Ethics and Sustainability to Informatics Engineering Students, An Almost 30 Years' Experience," Sustainability, vol. 12, no. 14, p. 5499, 2020, doi: $10.3390 /$ su 12145499.

[5] C. Fiesler. "Tech Ethics Curricula: A Collection of Syllabi." Medium. https://medium.com/@cfiesler/tech-ethics-curricula-a-collectionofsyllabi-3eedfb76be18 (accessed 25 de agosto de 2021, 2021).

[6] F. J. García-Peñalvo, A. Corell, V. Abella-García, and M. Grande-dePrado, "Online Assessment in Higher Education in the Time of COVID-19," Education in the Knowledge Society, vol. 21, Article 12, 2020, doi: 10.14201/eks.23086.

[7] H. Fardoun, C. S. González-González, C. A. Collazos, and M. Yousef, "Exploratory Study in Iberoamerica on the Teaching-Learning Process and Assess-

ment Proposal in the Pandemic Times," Education in the Knowledge Society, vol. 21, Article 17, 2020, doi: 10.14201/eks.23437.

[8] F. J. García-Peñalvo, A. Corell, R. Rivero-Ortega, M. J. RodríguezConde, and N. Rodríguez-García, "Impact of the COVID-19 on Higher Education: An Experience-Based Approach," in Information Technology Trends for a Global and Interdisciplinary Research Community, F. J. García-Peñalvo Ed. Hershey, PA, USA: IGI Global, 2021, pp. 1-18. 